\title{
Covalent functionalization of molybdenum disulfide by chemically activated diazonium salts
}

Received 00th January 20xx, Accepted 00th January 20xx DOI: $10.1039 / x 0 x \times 00000 x$

\author{
Lakshya Daukiya, ${ }^{1}$ Joan Teyssandier, ${ }^{1}$ Samuel Eyley, ${ }^{2}$ Salim El Kazzi, ${ }^{3}$ Miriam Candelaria Rodríguez \\ González, ${ }^{1}$ Bapi Pradhan, ${ }^{1}$ Wim Thielemans, ${ }^{2}$ Johan Hofkens ${ }^{1}$ and Steven De Feyter*1
}

\begin{abstract}
Covalent functionalization is one of the most efficient ways to tune the properties of layered materials in a highly controlled manner. However, molecular chemisorption on semiconducting transition metal dichalcogenides remains a delicate task due to the inertness of their surface. Here we perform covalent modification of bulk and single layer molybdenum disulfide $\left(\mathrm{MoS}_{2}\right)$ using chemical activation of diazonium salts. A high level of control over the grafting density and yield on MoS 2 basal plane can be achieved by this approach. Using scanning probe microscopies and X-ray photoelectron spectroscopy we prove the covalent functionalization of $\mathrm{MoS}_{2}$.
\end{abstract}

\section{Introduction}

Transition metal dichalcogenides (TMDs) are a class of layered materials that gained considerable interest of the research community over the past few years due to their astounding physical and chemical properties. ${ }^{1,2}$ Layered TMDs are composed of an atomic layer of transition metal $(\mathrm{M})$ sandwiched between two atomic layers of chalcogen $(X)$. These TMD sheets are stacked together via van der Waals forces. TMDs exist as different polymorphs depending on the co-ordination of the metal atoms, leading to a trigonal prismatic (hexagonal, $H$ ), octahedral (tetragonal, $T$ ) and a distorted phase $\left(T^{\prime}\right)$. The $d-$ orbital count and the co-ordination of the metal atoms influence the electronic band structure of TMDs. Therefore, the bandgap and the electronic band structure varies depending on the transition metal atoms. Few layer and monolayer TMDs exhibit different properties compared to their bulk counterparts because of quantum confinement and surface effects. Among all the members of TMDs, the $2 \mathrm{H}$ phases based on molybdenum and tungsten are of particular interest due to their bandgap which spans the visible and the infrared regions of the electromagnetic spectrum, therefore making these TMDs highly promising for a number of (opto)-electronic applications. ${ }^{1,3,4}$ Monolayer $\mathrm{MoS}_{2}$, one of the most studied TMDs, has a direct bandgap of around $1.9 \mathrm{eV}$ and a high charge carrier mobility that can reach $500 \mathrm{~cm}^{2} \mathrm{~V}^{-1} \mathrm{~s}^{-1}$. $^{2}$

Molecular approaches have extensively been explored over the past few years as an effective method to fine-tune the properties of $2 \mathrm{D}$ materials. ${ }^{5} \mathrm{~A}$ vast library of molecules provides a lot of room to introduce desired functionalities in 2D materials. Among the various approaches established, covalent functionalization of 2D materials is one of the most interesting ones due to its impact and stability. Co-

${ }^{1}$ Division of Molecular Imaging and Photonics, Department of Chemistry, KU LeuvenUniversity of Leuven, Celestijnenlaan, 200 F, 3001 Leuven, Belgium

${ }^{2}$ Sustainable Materials Lab, Department of Chemical Engineering, KU Leuven Campus Kortrijk, Etienne Sabbelaan 53, 8500 Kortrijk, Belgium

${ }^{3}$ imec, Kapeldreef 75, B-3001 Leuven, Belgium ${ }^{\dagger}$

Corresponding author: steven.defeyter@kuleuven.be

Electronic Supplementary Information (ESI) available. See DOI: 10.1039/x0xx00000x valent functionalization of graphene by a number of reactive molecules has shown to tune its electronic properties. ${ }^{6-9}$ Similar functionalization approaches are predicted to result in tuning the electronic properties of TMDs as well for a broad range of applications. ${ }^{10,11}$ Over the past few years, different strategies for the functionalization of TMDs have been developed which focus on improving the processability of the nanosheets in solution, studying the impact of sulfur vacancies, and enhancing their catalytic properties. ${ }^{12}$ Various reactive molecules were used such as organohalides ${ }^{13}$, thiols, ${ }^{14,15}$ diazonium salts ${ }^{16-19}$ and maleimide derivatives. ${ }^{20}$ Initial attempts of covalent functionalization of $\mathrm{MoS}_{2}$ by organohalides and diazonium salts were carried out via intercalation and subsequent liquid-phase exfoliation of $\mathrm{MoS}_{2}$ nanosheets followed by the covalent reaction with the organohalides or diazonium salts. ${ }^{13}, 17$ This approach involves the transformation of the semiconducting $2 \mathrm{H}$ phase of $\mathrm{MoS}_{2}$ to the more reactive, metallic $1 \mathrm{~T}$ phase. Another popular method of functionalizing substrate supported $\mathrm{MoS}_{2}$ is the attachment of thiol molecules, which react with $\mathrm{MoS}_{2}$ at the $S$ vacancy sites via a sulfur substitution route. ${ }^{14,21}$ However, this method requires pre-existing $S$ vacancies and the nature of the bond formed is still under debate. ${ }^{15}$, 22-24

Among the methods for functionalization of 2D materials and particularly TMDs, the one involving the grafting of aryl radicals generated by the reduction of diazonium salts ${ }^{25}$ is definitely a promising one. Aryl radicals can covalently graft on a defect-free surface. Furthermore, the choice between a large variety of functional aryl radical derivatives is attractive and broadens the application scope of TMDs.

However, in order to effectively tune the electronic properties of TMDs such as doping levels and to make TMD-based sensing devices for specific applications which require special receptors, it is important to explore methods by which TMDs can be functionalized with a sufficiently high density by a wide range of diazonium species. Due to the intrinsic bandgap in Mo and W based TMDs ( $2 \mathrm{H}$ phase is not an effective electrode), the covalent functionalization by electrochemical activation of diazonium ions is not straightforward. In order to overcome this limitation, spontaneous grafting of aryl radicals was explored. It has been shown recently that bulk and chemical vapor deposition (CVD)-grown $\mathrm{MoS}_{2}$ can be readily functionalized in their 

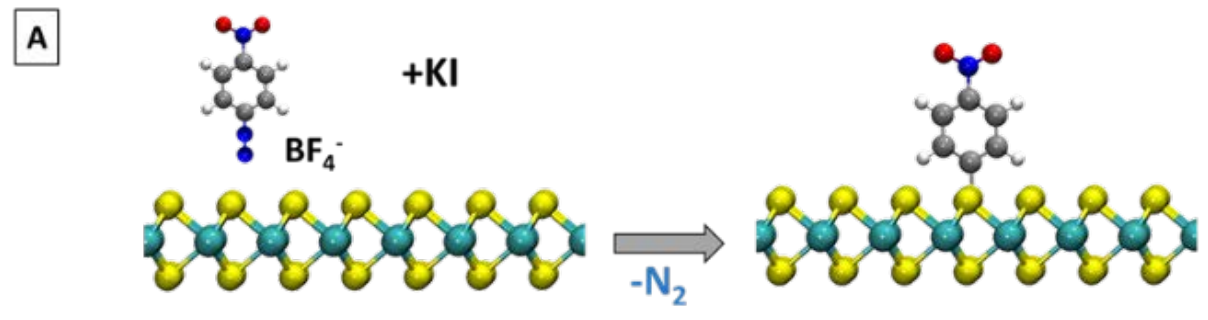

$1.5 \mathrm{~nm}$

$1.5 \mathrm{~nm}$

$1.5 \mathrm{~nm}$
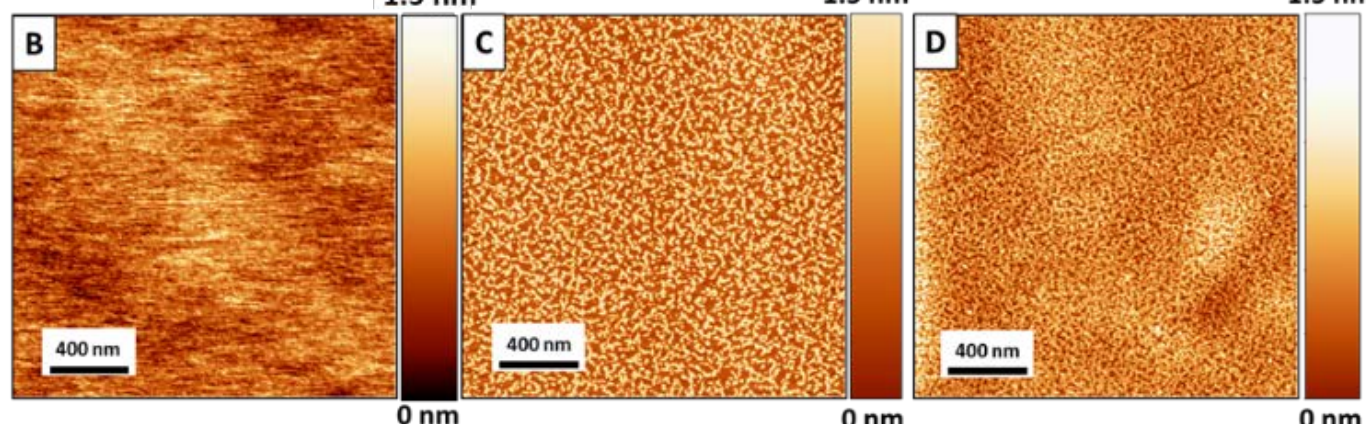

Figure 1) A) Schematic of reaction between 4-NBD and MoS2 lattice. AFM topography images of B) pristine bulk MoS2 and C) bulk MoS 2 after functionalization with $0.05 \mathrm{mM}$ 4-NBD/KI. D) shows an increased density of features on $\mathrm{MoS}_{2}$ surface after longer (10 min) reaction time. RMS roughness is (b) $0.46 \mathrm{~nm}$ (c) $0.43 \mathrm{~nm}$ and (d) $0.23 \mathrm{~nm}$.

pristine $2 \mathrm{H}$ form by spontaneous reaction of aryl radicals obtained from spontaneous decomposition of diazonium salts such as 4-nitrobenzenediazonium and 4-carboxybenzenediazonium. ${ }^{16}$ This method is of interest as there is no pre-functionalization process required. The aryl radicals graft on the $S$ atoms of the $\mathrm{MoS}_{2}$ lattice, while the grafting process is assisted by a small number of defects, since $S$ vacancy sites act as nucleation centres for the initial functionalization. The limitation of spontaneous grafting is lack of control of the grafting density. This process is also limited to those diazonium salts that are reactive enough to undergo spontaneous grafting.

To circumvent the limitations of electrochemical activation and spontaneous grafting, in situ chemical activation of diazonium salts resulting in formation of aryl radicals could be used to functionalize semiconducting TMDs. Interestingly, a similar approach, where alkyl halides were chemically reduced, has recently been used to functionalize $1 T^{\prime}-\mathrm{MoS}_{2} \cdot{ }^{26}$ It has been shown earlier that, in the case of diazonium salts, chemical activation via ascorbic acid or iron powder can form aryl radicals for covalent functionalization of a wide range of substrates. ${ }^{27-29}$ Even before that, this approach was used to perform aryl-aryl coupling in solution, for instance using iodide to induce the reduction of aryl diazonium salts, obtaining quantitative yield of biaryls. ${ }^{30}$ Using a similar strategy our group has recently carried out a detailed study on the covalent functionalization of graphene and graphite surfaces by 4-nitrophenyl (4-NP) formed via chemical activation of 4-nitrobenzenediazonium tetrafluoroborate (4-NBD) ions using potassium iodide (KI). A detailed account can be found elsewhere. ${ }^{31}$

In this contribution, we demonstrate that using chemical activation it is possible to efficiently decorate the $\mathrm{MoS}_{2}$ basal plane, starting from 4-NBD, the most popular diazonium salt for surface functionalization. Compared with spontaneous grafting, the grafting density can be controlled and a higher density is reached. Furthermore, dendritic growth leading to thick film formation is avoided. All of this is achieved for much lower reaction times and with lower reagent concentrations, as documented by scanning probe microscopy (SPM) and spectroscopy techniques. Chemical activation is not limited to just one reducing agent (KI), or one diazonium derivative. Other activators such as iron powder may as well be used to activate and graft diazonium salts with a very low reactivity.

\section{Results and Discussion}

We begin by grafting chemically activated 4-nitrobenzene diazonium (4-NBD) ions on bulk and CVD grown $\mathrm{MoS}_{2}$ substrates. 4-NBD was chosen because of its popularity and to understand the role of chemical activation in improving the overall functionalization efficiency. The activation of 4-NBD into 4-nitrophenyl (4-NP) radicals was carried out using the same method reported in ref. 27. Aqueous solutions of 4-NBD and KI were mixed in a 1:1 ratio immediately followed by drop casting on $\mathrm{MoS}_{2}$. Figure $1 \mathrm{~A}$ shows the schematic representation of the reaction between $\mathrm{MoS}_{2}$ and 4-NP, generated from 4-NBD using $\mathrm{KI}$. The iodide anion from $\mathrm{KI}$ acts as the reducing agent in this reaction. SPM and spectroscopy measurements confirmed the covalent functionalization of $\mathrm{MoS}_{2}$ substrate by this method.

\section{Atomic force microscopy}

Figure 1 B \& C show the atomic force microscopy (AFM) images of bulk $\mathrm{MoS}_{2}$ before and after exposure to $0.05 \mathrm{mM}$ solution of 4-NBD in aqueous solution with $0.05 \mathrm{mM}$ of KI. For pristine bulk $\mathrm{MoS}_{2}$ the AFM topographic image reveals a clean surface. After exposure to 4NP-radicals the surface is highly modified as shown in Figure $1 \mathrm{C}$. Numerous randomly scattered features as a layer on the $\mathrm{MoS}_{2}$ surface are observed. The layer thickness is rather uniform $(1.2 \pm 0.2 \mathrm{~nm})$, as determined by using empty areas already present on the surface as a reference, or by inducing them by locally removing molecules using an AFM based nanolithography method (nanoshaving) (Figure SI 1 and $\mathrm{SI} 2 \mathrm{~A} \& \mathrm{~B}$ ). It was also observed that the number of these features depends on the concentration of the diazonium salt as well as the reaction time with the $\mathrm{MoS}_{2}$ surface. At $0.05 \mathrm{mM}$, the coverage increased from $35 \pm 5 \%$ after 1 min to $78 \pm 6 \%$ after 10 min of reaction (Figure $1 \mathrm{C}$ and $\mathrm{D}$ ) (see methods section for details about measuring the coverage). Upon increasing the concentration to [4- 

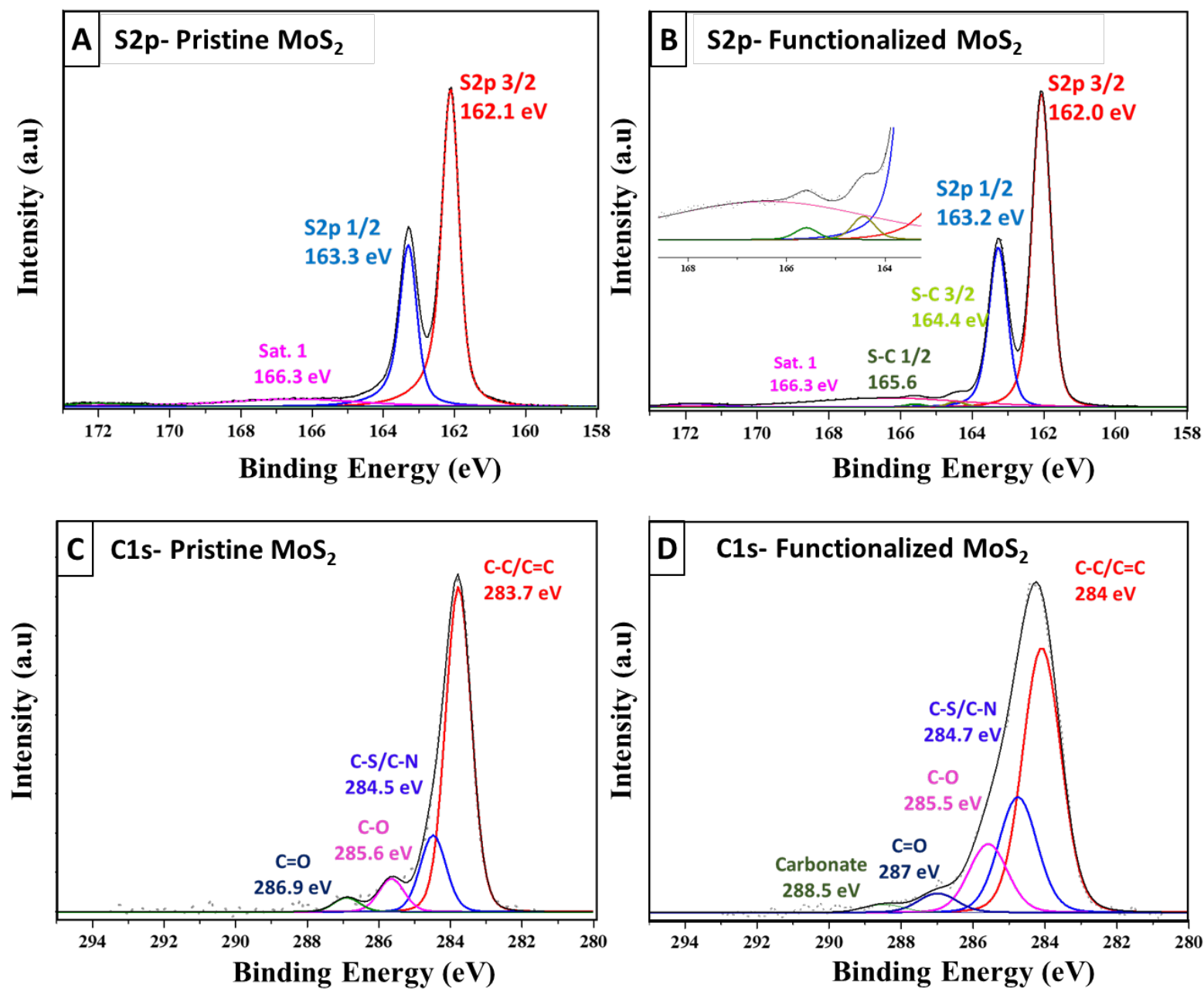

Figure 2 : S $2 p$ core level spectra on CVD MoS 2 on sapphire A) shows the typical S $2 p$ doublet with $1.2 \mathrm{eV}$ difference due to spin orbit splitting. B) After functionalization we observe an additional doublet towards higher binding energy located at $164.4 \mathrm{eV}$ and $165.6 \mathrm{eV}$ which is attributed to C-S interactions resulting from covalent bonding of 4-NP molecules with the S-atoms of $M_{0} S_{2}$ surface. C) and D) show the C 1s spectra before and after functionalization. For pristine $\mathrm{MoS}_{2}$ the components of $\mathrm{C} 1 \mathrm{~s}$ spectra mostly correspond to atmospheric contaminants. After functionalization the $\mathrm{C} 1 \mathrm{~s}$ peak is broadened with $\mathrm{C}-\mathrm{C} / \mathrm{C}=\mathrm{C}$ component resulting from phenyl rings of 4-NP and an increased area of C-S resulting from covalent grafting of 4-NP with $\mathrm{MoS}_{2}$.

$\mathrm{NBD}]=[\mathrm{KI}]=0.25 \mathrm{mM}$ we observed a very high coverage of these features on the surface as shown in Figure SI 2 A\&B. The surface was already almost fully covered after $1 \mathrm{~min}$ and did not evolve noticeably for longer exposure times ( $97 \pm 4 \%$ after $1 \mathrm{~min}$ and $93 \pm 9 \%$ after $10 \mathrm{~min})$. A significant effect of the chemical activation of 4-NBD is that it limits drastically the layer thickness. While the thickness produced by the chemical activation of 4-NBD is limited to no more than $2 \mathrm{~nm}$, spontaneous grafting of the same molecule produces uncontrolled vertical growth of the film (Figure SI 2E\&F). For instance, after $1 \mathrm{~min}$ of exposure to a $0.25 \mathrm{mM}$ solution of 4-NBD only, an average thickness of $1.1 \pm 0.6 \mathrm{~nm}$ was measured $(1.8 \pm 0.9 \mathrm{~nm}$ in the presence of $\mathrm{KI}$ ). After $10 \mathrm{~min}$ in a $0.25 \mathrm{mM}$ solution of 4-NBD only, the thickness reached $4.9 \pm 0.4 \mathrm{~nm}(2.0 \pm 1.0 \mathrm{~nm}$ in the presence of KI) (Figure SI $2 \mathrm{~F}$ ). We have also carried out similar functionalization experiments on $\mathrm{MoS}_{2}$ grown on sapphire by CVD. The CVD-MoS 2 was used to confirm the feasibility of the chemical activation protocol on single to few layer $\mathrm{MoS}_{2}$ as well as for the facility that CVD grown MoS 2 provides for spectroscopic studies. The AFM images on pristine and 4NP functionalized (generated in situ by KI activation method) CVD $\mathrm{MoS}_{2}$ are shown in Figure SI 3. AFM image of pristine CVD-MoS shows a number of hexagonal domains with some smaller triangular domains on top of it. The hexagonal domains are attributed to the second layer of $\mathrm{MoS}_{2}$ on top of a fully homogeneous first layer. The secondary domains are formed as a result of uncontrolled defect sites in the first $\mathrm{MoS}_{2}$ layer which acts as nucleation sites. ${ }^{32}$ From the AFM images an overall increase in the surface coverage of features with an increase in the concentration of 4-NBD is also observed for CVD- $\mathrm{MoS}_{2}$. We also compared AFM images of 4-NP functionalized bulk and CVD-MoS , and similar features were observed on both bulk and CVD-MoS 2 as shown in figure SI 4. Therefore, the feasibility of the chemical activation protocol on monolayer to few layer $\mathrm{MoS}_{2}$ is also established. As extracted from the analysis of the AFM images, the thickness obtained for films grafted through chemical activation of the diazonium ions was again lower compared with the spontaneous grafting.

The difference in the growth of the layers, and the resulting layer thickness, on bulk as well as CVD-MoS 2 can be explained in terms of availability of radicals at the surface-solution interface. For the spontaneous grafting, radicals are provided by decomposition of the 4NBD ions after spontaneous reduction by the surface. ${ }^{33}$ This process 
takes place over time and the radical concentration increases gradually. Furthermore, the transfer of radicals to the already grafted molecules continues until the process reaches a limit because of the high thickness of the layer and the consequent poor electron transfer from the surface. In contrast, in case of the KI-based chemical activation of the diazonium ions, a number of side reactions can occur in solution, limiting the concentration of radicals in the proximity of the electrode. These side reactions in solution, which have been monitored previously by using UV-vis spectroscopy, decrease the availability of radicals at the surface-solution interface and, therefore, the attachment of new radicals to the already grafted molecules is limited. ${ }^{30}$

We have also carried out control experiments using only KI. The AFM measurements after drop casting $2.5 \mathrm{mM}$ of $\mathrm{KI}$ solution on $\mathrm{MoS}_{2}$
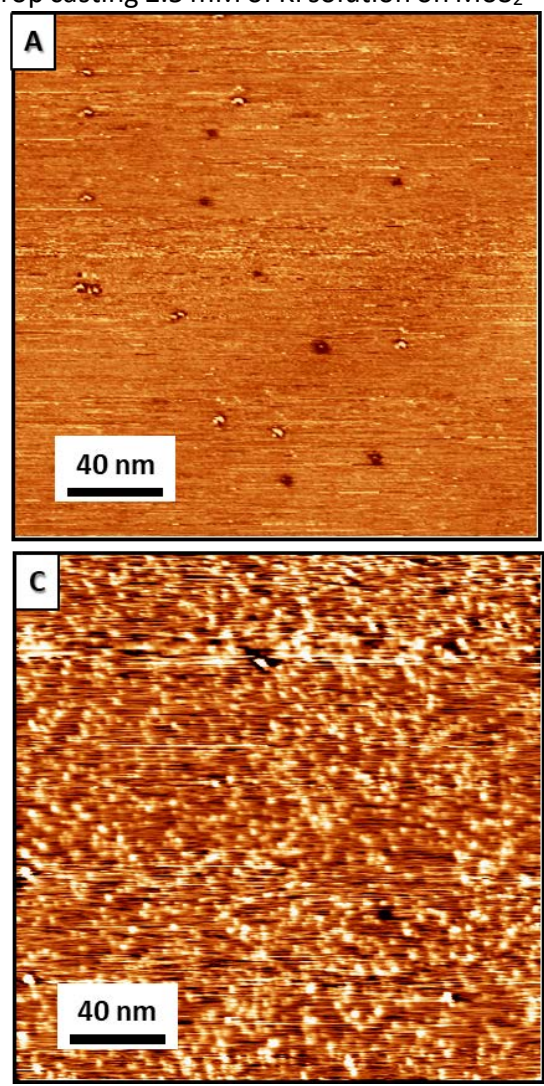

quantitatively evaluate the density of the molecules on $\mathrm{MoS}_{2}$. Therefore, we performed scanning tunnelling microscopy (STM) and X-ray photoelectron spectroscopy (XPS) measurements to confirm the presence of grafted molecules and quantitatively analyse the density of the grafted 4-NP. XPS was already successfully carried out for the characterization of NP-functionalized graphite and graphene ${ }^{34,35}$. We did XPS measurements on 4-NP functionalized CVD MoS 2 because, in the case of functionalized bulk $\mathrm{MoS}_{2}$, the signal from the top layer is swamped by the bulk $\mathrm{MoS}_{2}$ signal making it difficult to resolve the presence of $S-C$ bonds from the $S 2 p$ and $C 1$ s peaks. As the CVD$\mathrm{MoS}_{2}$ samples are mainly monolayers with only few patches of bilayer coverage, it is expected that any modification in the high-resolution spectra will be clearly observed. We used CVD-MoS 2 functionalized with $0.5 \mathrm{mM}$ of 4-NBD for $5 \mathrm{~min}$ for XPS measurements. The
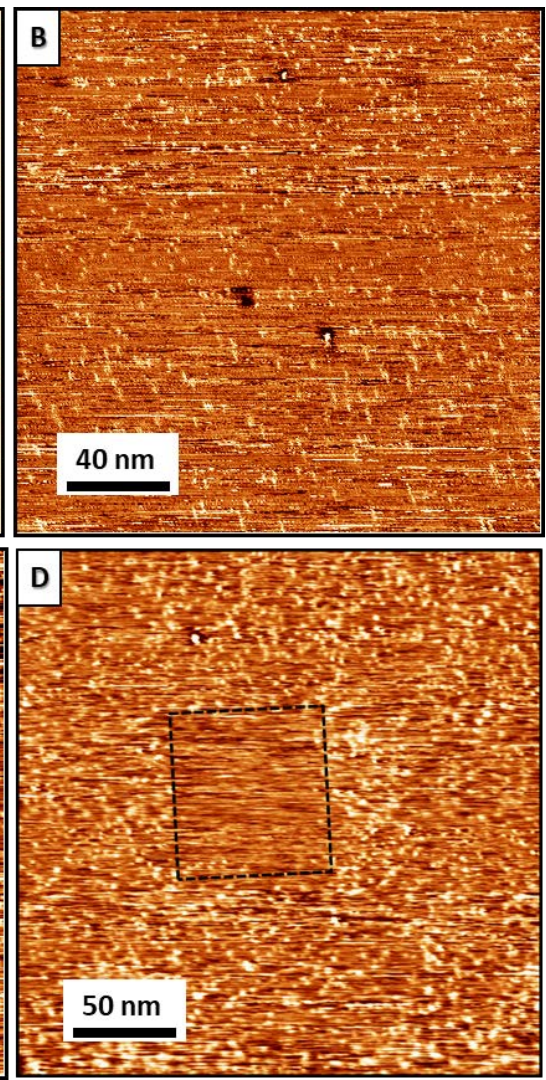

Figure 3: STM images of A) Pristine bulk $\mathrm{MoS}_{2}$ surface showing a clean surface with few dark spots corresponding to point defects (imaging parameters: $I_{\text {set }}=0.09 \mathrm{nA}, \mathrm{V}_{\text {bias }}=-1 \mathrm{~V}$ ). B) STM image of $\mathrm{MoS}_{2}$ functionalized by $0.5 \mathrm{mM}$ 4-NBD using spontaneous grafting protocol (imaging parameters: $I_{\text {set }}=0.06 \mathrm{nA}, \mathrm{V}_{\text {bias }}=-1.2 \mathrm{~V}$ ). C) STM image of $\mathrm{MoS}_{2}$ functionalized by $0.5 \mathrm{mM}$ 4-NBD using KI activation protocols (imaging parameters: $\left.I_{\text {set }}=0.06 \mathrm{nA}, V_{\text {bias }}=-0.9 \mathrm{~V}\right)$. D) STM image in molecules in the central area have been removed by STM-based lithography (nanoshaving) (imaging parameters: $I_{\text {set }}=0.07 \mathrm{nA}, V_{\text {bias }}=-1 \mathrm{~V}$ ). All the images were acquired at liquid/solid interface using 1-phenyloctane.

are shown in Figure SI 5. Note that this concentration is ten times higher than the highest concentration used for NBD grafting. These AFM images show some sparsely distributed features for bulk $\mathrm{MoS}_{2}$ and almost no features for CVD-MoS 2 . This suggests that the surface coverage observed from AFM images of figure 1 and Figure SI 2 \& 3 is predominantly due to NBD molecules and only to a small fraction of KI residues. The AFM results give insight into the surface morphology of functionalized $\mathrm{MoS}_{2}$ and its dependence on the experimental conditions.

\section{X-ray photoelectron spectroscopy}

AFM does not distinguish between chemisorbed and physisorbed material. Additionally, due to spatial resolution issues it is difficult to wide scan XPS spectra of pristine CVD-MoS 2 and 4-NP functionalized CVD- $\mathrm{MoS}_{2}$ are shown in Figure SI 7A.

The high resolution $S 2 p$ and $C$ 1s core level spectra are shown in Figure 2. The details of experimental conditions are mentioned in the experimental section. The dotted black curve is the experimental data and the coloured curves show deconvoluted peaks. As a result of spin-orbit interaction $S 2 p$ spectra are split in two components, $S$ $2 \mathrm{p}_{3 / 2}$ and $S 2 \mathrm{p}_{1 / 2}$ separated by $1.2 \mathrm{eV}$, with an intensity ratio around 0.5 as shown in Figure $2 \mathrm{~A} \& \mathrm{~B}$. This is in agreement with earlier reports. ${ }^{36} \mathrm{~A}$ broad feature at higher binding energy around $166.3 \mathrm{eV}$ corresponds with surface plasmon losses ${ }^{37}$. After functionalization the $S 2 p$ spectra show a slight modification (Figure 2B). Along with the doublet corresponding to the unmodified $\mathrm{S}$ atoms we also observed a small doublet at higher binding energy. The position of this 
new doublet peak matches the S-C binding energy, ${ }^{38}$ which confirms the reaction of 4-NP radicals with the $\mathrm{S}$ atoms of $\mathrm{MoS}_{2}$ layer. The intensity of the S-C peak is much lower compared to the peak intensity of the unmodified $\mathrm{S}$ atoms of $\mathrm{MoS}_{2}$. The percentage area composition of S-C peak was only $1.5 \%$ of the overall S $2 p$ signal. This observation can be explained by the fact that not all the surface $S$ atoms react with the 4-NP radicals. This is in line with the grafting density we calculated from the STM images (see below).

C 1s spectra also give confirmation of covalent grafting. Before functionalization, peaks in the $C 1 \mathrm{~s}$ are attributed to environmental impurities on the surface of $\mathrm{MoS}_{2}$ as shown in Figure 2C. The peak at $283.7 \mathrm{eV}$ corresponds to the aliphatic/aromatic carbon impurities while the peaks at higher binding energy can be attributed to the C$\mathrm{O}$ and $\mathrm{C}=\mathrm{O}$. The additional peak at $284.5 \mathrm{eV}$ can be attributed to $\mathrm{C}-\mathrm{S}$ and/or $\mathrm{C}-\mathrm{N}$ arising from the contamination during the growth process of $\mathrm{MoS}_{2}$ films via CVD. After functionalization, we observe that the peak at $284 \mathrm{eV}$ increases which can be attributed to additional C atoms of the grafted 4-NP molecules. The peak corresponding to C-S extending more than $200 \times 200 \mathrm{~nm}^{2}$. A few dark pits were observed on the surface (Figure $3 \mathrm{~A}$ ), which correspond to the presence of sulfur vacancies in the subsurface layer of $\mathrm{MoS}_{2}$. Such defects are common in $\mathrm{MoS}_{2}$ layers and have been studied in detail earlier. ${ }^{14}$ In order to compare the spontaneous grafting of 4-NP radicals on $\mathrm{MoS}_{2}$ with grafting of chemically activated 4-NP radicals, we functionalized $\mathrm{MoS}_{2}$ with the similar concentration of 4-NBD for the same reaction time ( $0.5 \mathrm{mM}$ for 5 minutes). Figure $3 \mathrm{~B}$ and $3 \mathrm{C}$ show STM images acquired on $\mathrm{MoS}_{2}$ functionalized by spontaneously grafted and chemically generated 4-NP radicals, respectively.

In Figure 3B the average feature area is about $2.0 \pm 0.3 \mathrm{~nm}^{2}$ which roughly translates to a feature diameter of $1.6 \pm 0.3 \mathrm{~nm}$. Similar measurements on surfaces formed by the KI activation process (Figure 3 C) gave an average feature area around $2.2 \pm 0.3 \mathrm{~nm}^{2}$ and feature diameter of $1.9 \pm 0.2 \mathrm{~nm}$ (see Figure SI 7 for line profile analysis of features observed by STM imaging). The average apparent height of the features in figure $2 B$ was around $1.4 \pm 0.2 \mathrm{~nm}$ at $-1 \mathrm{~V}$ bias voltage applied to the sample. The similarities in the dimensions of the

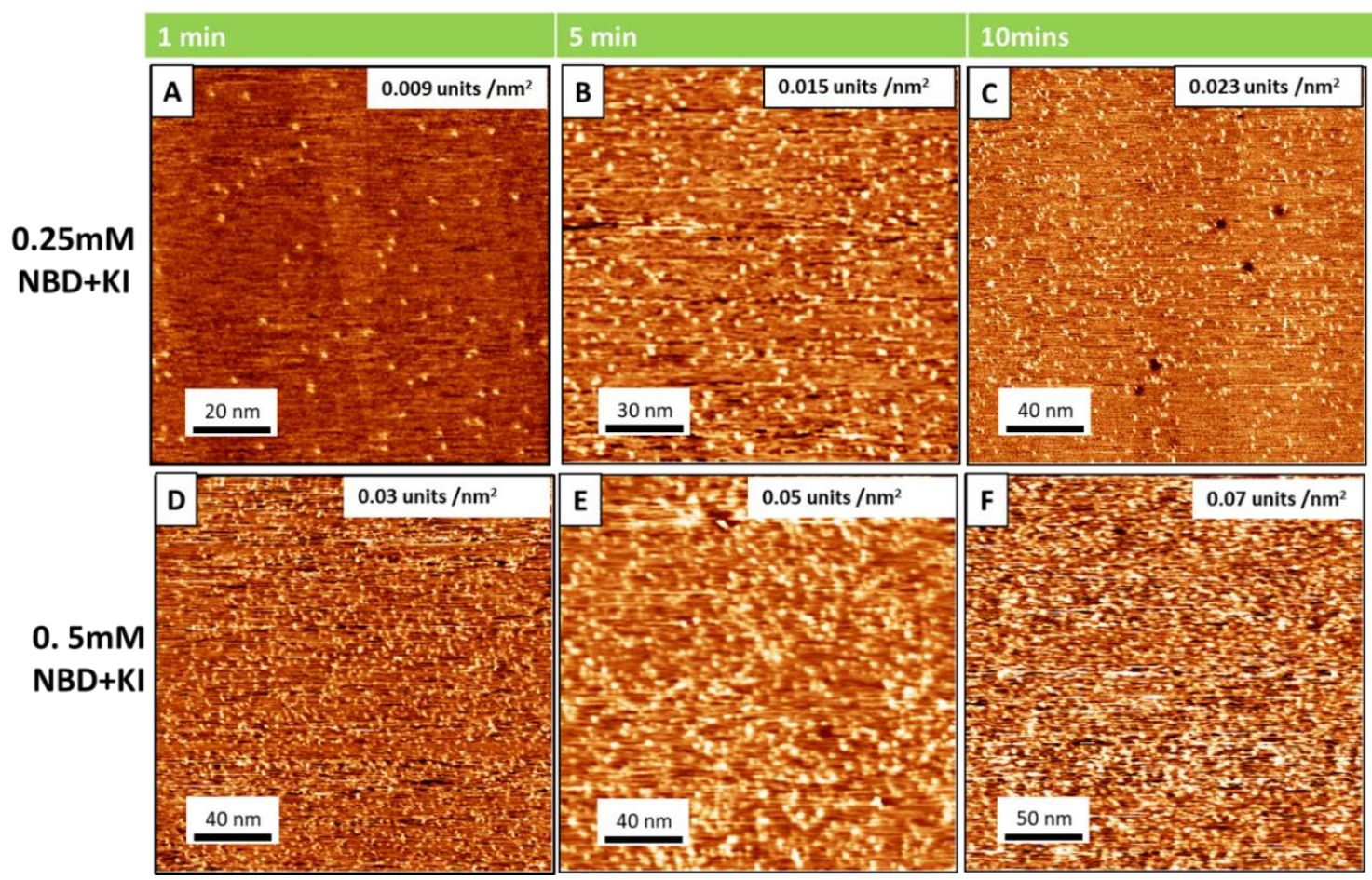

Figure 4: STM images on 4-NP functionalized bulk $\mathrm{MoS}_{2}$ showing the role of reaction time and concentration of reactants towards the overall grafting density of 4-NP on $\mathrm{MoS}_{2}$. The STM images were acquired at liquid solid interface using $1 \mathrm{PO}$ as solvent. Imaging parameters are a) $I_{\text {set }}$ $=0.05 \mathrm{nA}, V_{\text {bias }}=-0.8 \mathrm{~V}$, b) $\left.\left.\left.I_{\text {set }}=0.04 \mathrm{nA}, V_{\text {bias }}=-0.8 \mathrm{~V}, \mathrm{c}\right) I_{\text {set }}=0.07 \mathrm{nA}, V_{\text {bias }}=-0.8 \mathrm{~V}, \mathrm{~d}\right) I_{\text {set }}=0.06 \mathrm{nA}, V_{\text {bias }}=-1 \mathrm{~V}, e\right) I_{\text {set }}=0.06 \mathrm{nA}, V_{\text {bias }}=$ $-0.9 \mathrm{~V}, \mathrm{f}) \mathrm{I}_{\text {set }}=0.07 \mathrm{nA}, \mathrm{V}_{\text {bias }}=-1.1 \mathrm{~V}$.

at $284.7 \mathrm{eV}$ increases suggesting formation of covalent bonds between $\mathrm{S}$ atoms of $\mathrm{MoS}_{2}$ and 4-NP radicals (Figure 2D).

The Mo 3d spectra (Figure SI 6B) taken before and after the functionalization reactions did not show any modification as expected because the Mo layer is sandwiched between the $S$ layers and does not take part in the reactions. Additionally, we can conclude from the $\mathrm{MoS}_{2}$ spectra that there is no oxidation of the Mo atoms caused by the functionalization reactions as the peak positions do not change significantly.

\section{Scanning tunnelling microscopy}

Figure 3 shows STM images of the $\mathrm{MoS}_{2}$ surface at the liquid/solid interface before and after functionalization by 4-NP radicals. Prior to functionalization the surface of $\mathrm{MoS}_{2}$ looks clean, with large terraces features observed in Figure $3 \mathrm{~B}$ and $3 \mathrm{C}$, i.e. with and without $\mathrm{KI}$ activation, suggests that these features can be interpreted as covalently bound 4-NP (single) molecules, and not as KI residues. Also, these features are similar in dimensions with the 4-NP species grafted on graphene and HOPG as reported earlier which further supports the argument that these are 4-NP molecules grafted on $\mathrm{MoS}_{2}{ }^{7}$

For chemically activated 4-NP radicals, the density of grafting on $\mathrm{MoS}_{2}$ is much higher ( 0.05 molecules $/ \mathrm{nm}^{2}$ ) in comparison to spontaneously grafted 4-NP radicals $\left(0.012\right.$ molecules $\left./ \mathrm{nm}^{2}\right)$. This increase in surface coverage with concentration and time is also observed by the AFM measurements (Table SI1). We tried to mechanically remove the molecules from a small area $\left(70 \times 70 \mathrm{~nm}^{2}\right)$ by applying high tunnelling current and very low bias similar to the method used for removing grafted 4-NP molecules from HOPG surface. ${ }^{7}$ We observed 
that after nanoshaving (Figure 3D), the area (black dotted line) is completely free of any features while outside this area bright features similar to those in Figure $3 \mathrm{C}$ can be observed. This suggests that, similar to what was observed on graphite and graphene, molecules can be mechanically removed after grafting to create nanopatterns of (un)functionalized $\mathrm{MoS}_{2}$. Similar STM measurements on 4NP functionalized CVD-MoS 2 (both spontaneous and chemical activated grafting), turned out unsuccessful, presumably due to inhomogeneity of the $\mathrm{MoS}_{2}$ layer, affecting the electrical contact adversely.

The density of covalent functionalization of $\mathrm{MoS}_{2}$ as a function of experimental conditions such as reaction time and concentration of 4-NBD was also quantified. Aqueous solutions of 4-NBD and KI were first prepared separately with a 1:1 concentration ratio and then mixed together followed by drop casting on bulk $\mathrm{MoS}_{2}$. We used two different solutions of 4-NBD $+\mathrm{KI}, 0.25 \mathrm{mM}$ and $0.5 \mathrm{mM}$, that were drop cast on $\mathrm{MoS}_{2}$ for 1, 5 and 10 minutes each. Representative STM images acquired on these six $\mathrm{MoS}_{2}$ samples are shown in Figure 4. An increase in the overall density of the features with increasing reaction duration and concentration of 4-NDB and KI solution can be clearly observed from the STM images. We observed that for higher concentration of 4-NBD molecules the size of the features also increased and therefore the features appeared as clusters in the STM images. The density of features, which was plotted against the reaction time for each concentration, (Figure SI 8) also showed a consistent increase.

\section{Raman spectroscopy}

Raman spectroscopy measurements were carried out to determine the influence of covalent grafting of 4-NP molecules on the structural properties of $\mathrm{MoS}_{2}$. The typical Raman spectrum of $2 \mathrm{H}-\mathrm{MoS}_{2}$ has two prominent Raman active modes at approximately $383 \mathrm{~cm}^{-1}$ and 408 $\mathrm{cm}^{-1}$ arising from $E_{2 \mathrm{~g}}^{1}$ and $A_{1 \mathrm{~g}}$ vibrational modes at the $\Gamma$ point of the Brillouin zone. ${ }^{39}, 40 \mathrm{In}$ addition the peak at $450 \mathrm{~cm}^{-1}$ corresponds to the second order longitudinal acoustic phonon (2LA (M)). Figure 5A shows the Raman spectra of $\mathrm{MoS}_{2}$ before (black) and after (red) functionalization with [4-NBD]=[KI] $=2.5 \mathrm{mM}$ for a reaction time of 5 minutes. The spectra shown in this figure were acquired for $10 \mathrm{sec}-$ onds each, and repeated 6 times. A short irradiation time was chosen to prevent localized heating of the $\mathrm{MoS}_{2}$ surface which could potentially result in desorption of grafted 4-NP. For the CVD MoS samples, $A_{1 \mathrm{~g}}$ and $\mathrm{E}^{1}{ }_{2 \mathrm{~g}}$ modes were located at a frequency difference of $25 \mathrm{~cm}^{-}$ 1 i.e. $8 \mathrm{~cm}^{-1}$ lower when compared to bulk $\mathrm{MoS}_{2}$, which confirming the monolayer nature of $\mathrm{CVD}-\mathrm{MoS}_{2}$. Comparing the spectra before

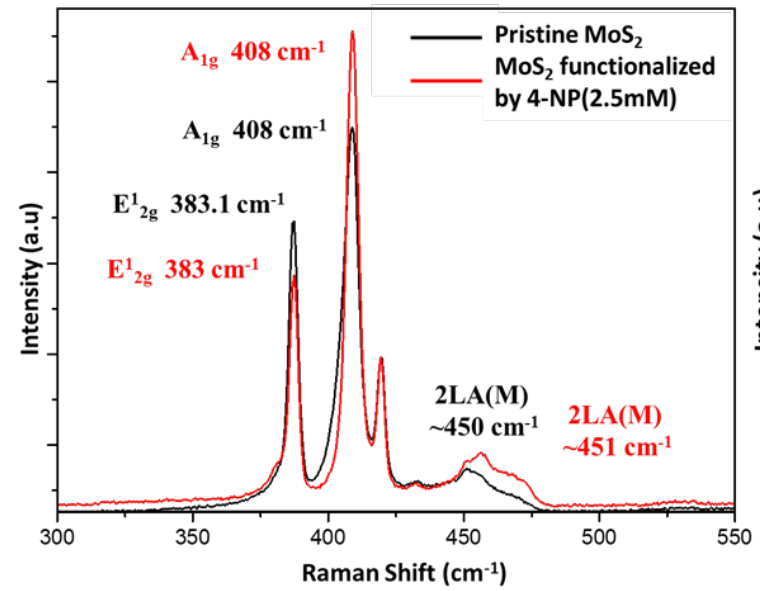

and after functionalization, we observed an overall change in the intensity ratio of $\mathrm{E}_{2 \mathrm{~g}}^{1}$ and $\mathrm{A}_{1 \mathrm{~g}}$, which was 0.77 for pristine CVD-MoS compared to 0.51 for CVD-MoS functionalized by $2.5 \mathrm{mM}$ of NBD. The decrease in $\mathrm{E}^{1}{ }_{2 \mathrm{~g}} / \mathrm{A}_{1 \mathrm{~g}}$ intensity after functionalization is in agreement with an earlier report on diazonium functionalization of chemically exfoliated $1 \mathrm{~T}-\mathrm{MoS}_{2} \cdot{ }^{17} \mathrm{We}$ also observed slight modification of the 2LA (M) peak of CVD-MoS 2 after functionalization with 4-NP molecules. A slight increase in the intensity and FWHM was observed as compared to pristine CVD-MoS 2 . The increase in the overall intensity of the 2LA(M) peak is in contradiction with earlier reported studies. Knirsch et al. reported a higher intensity ratio of the $\mathrm{A}_{1 \mathrm{~g}}$ mode at 404 $\mathrm{cm}^{-1}$ to the $2 \mathrm{LA}(\mathrm{M})$ mode at $450 \mathrm{~cm}^{-1}$ for functionalized chemically exfoliated $\mathrm{MoS}_{2}$ (ce-MoS ) sheets as compared to non-functionalized ones. ${ }^{17}$ Whereas, in our studies we observed that for 4-NP functionalized CVD-MoS 2 the ratio of $A_{1 \mathrm{~g}}$ to $2 \mathrm{LA}(\mathrm{M})$ is lower when compared to pristine CVD-MoS 2 . We also observed that after functionalization a second component $\sim 457.6 \mathrm{~cm}^{-1}$ becomes more prominent. The opposite trend could come from the fact that Knirsch et al. used 1T$\mathrm{MoS}_{2}$ and the Raman spectroscopy was carried out using a different excitation laser ( $633 \mathrm{~nm}$ wavelength). Detailed studies on the Raman spectroscopy of covalently functionalized $\mathrm{MoS}_{2}$ will be necessary to gain a better understanding of the peak evolution.

\section{Photoluminescence}

We furthermore carried out photoluminescence $(\mathrm{PL})$ spectroscopy measurements to determine the influence of grafting on the electronic band structure of CVD-MoS 2 . Figure 5B compares the PL spectra of pristine CVD-MoS 2 and functionalized CVD-MoS 2 . After functionalization, the PL spectra of 4-NP grafted $\mathrm{MoS}_{2}$ is reduced significantly which could be a result of suppression of exciton recombination energy suggesting n-type doping of $\mathrm{MoS}_{2} \cdot{ }^{41}$ This observation is different from the common p-type doping induced by 4-NP groups in other 2D materials. ${ }^{42,43}$ The n-type doping can be attributed to KI. To verify this, we compared PL spectra of $\mathrm{MoS}_{2}$ functionalized by [4$\mathrm{NBD}]=[\mathrm{KI}]=0.5 \mathrm{mM}, 0.5 \mathrm{mM}$ of $\mathrm{KI}$ solution and $0.5 \mathrm{mM}$ of 4-NBD solution (Figure SI 9). The PL intensity of $[4-\mathrm{NBD}]=[\mathrm{KI}]=0.5 \mathrm{mM}$ functionalized $\mathrm{MoS}_{2}$ was almost similar to the PL intensity of $0.5 \mathrm{mM} \mathrm{KI}$ functionalized $\mathrm{MoS}_{2}$, whereas $\mathrm{MoS}_{2}$ functionalized by 4-NBD only showed a fairly comparable intensity as pristine $\mathrm{MoS}_{2}$. This confirms that the decrease in the PL intensity of $\mathrm{MoS}_{2}$ is due to n-type doping from KI. Recent studies have reported a change in the electronic band structure of $\mathrm{MoS}_{2}$ resulting from strong n-type doping due to KI. ${ }^{44}$ Such n-type doping may be undesirable for some applications

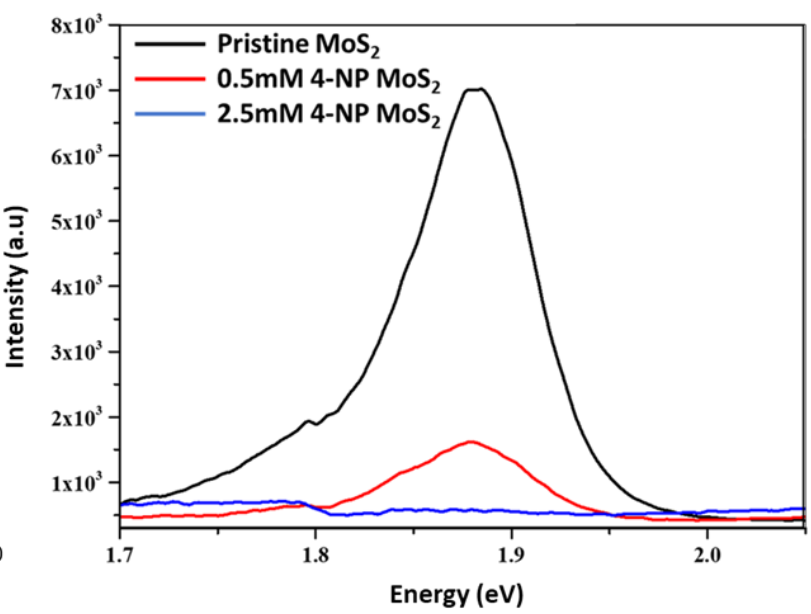

Figure 5: A) Raman spectra of pristine CVD-MoS 2 compared with CVD-MoS functionalized with $2.5 \mathrm{mM}$ of 4-NBD, the spectra were collected using a $532 \mathrm{~nm}$ He-Ne laser B) Comparison of photoluminescence spectra of pristine $\mathrm{MoS}_{2}$ with 4-NP functionalized MoS ${ }_{2}$, the PL spectra were carried out using excitation wavelength of $450 \mathrm{~nm}$ 
whereas it can be interesting for $\mathrm{MoS}_{2}$ based field effect transistors as it can lower the contact resistance and improve the charge carrier mobility. The choice of the reducing agent is thus crucial to tune the properties of functionalized TMDs. It becomes therefore important to investigate more of them, and identify those that do not interfere with the properties of TMDs, and those that can be used to tune these properties.

Note that this chemical activation approach is not restricted to 4NBD, though the choice of the activator will depend on the reactivity of the diazonium compound. For instance, 3,5-di-tert-butylphenyldiazonium (3,5-TBD) is less reactive. Following its in-situ formation from the aniline precursor 3,5-di-tert-butylphenylaniline (3,5-TBA) using a mixture of $\mathrm{NaNO}_{2}$ and $\mathrm{HCl}$, followed by adding $\mathrm{KI}$, no evidence of functionalization was observed. However, upon using the stronger reducing Fe powder instead of $\mathrm{KI}$, modification of the $\mathrm{MoS}_{2}$ surface was observed suggesting covalent grafting of 3,5-di-tert-butylphenyl (3,5-TBP) radicals (Figure SI 10).

\section{Conclusion}

In this study we have demonstrated the functionalization of TMDs by diazonium chemistry. We have shown the role of in situ chemical activation of diazonium salts by suitable reductants to functionalize $\mathrm{MoS}_{2}$ and achieved a precise control over the degree of functionalization. AFM and STM confirmed that the density of features, which we attributed to 4-NP molecules, can be significantly enhanced by using the chemical activation protocol. We have also provided proof of the covalent nature of the grafting using XPS. Raman spectroscopy and photoluminescence studies on functionalized samples showed strong modification compared to pristine $\mathrm{MoS}_{2}$ samples. We have also established that chemical activation is promising method for covalent grafting of diazonium salts of different reactivity. This work opens the way to the further investigation of the influence of controlled diazonium grafting on $\mathrm{MoS}_{2}$.

\section{Experimental Methods}

Materials. High-purity water (Milli-Q, Millipore, $18.2 \mathrm{M} \Omega \mathrm{cm}$, TOC < $3 \mathrm{ppb}$ ) was used for preparation of the aqueous solutions. Bulk crystalline $\mathrm{MoS}_{2}$ samples were purchased from SPI supplies. 4-Nitrobenzenediazonium (NBD) tetrafluoroborate $(97 \%)$, potassium iodide (KI) $(99.9 \%)$ and 1-phenyloctance were purchased from Sigma-Aldrich, all compounds were used as received. 3,5-Bis-tert-butylbenzenediazonium (3,5-TBD) was synthesized from the aniline precursor immediately prior to reduction. 3,5-Bis-tert-butylaniline (98\%) was purchased from TCl-Tokyo Chemical Industry Co., Ltd. and used without further purification. Iron powder $(99.9 \%)(<10 \mu \mathrm{m})$ was purchased from Sigma-Aldrich and was used as received.

CVD- $\mathrm{MoS}_{2}$ was grown on 2-inch c-plane oriented sapphire substrate. The substrate was annealed at $1175^{\circ} \mathrm{C}$ under $\mathrm{O}_{2}$ environment for 1 hour. Following this, the sapphire substrates were transferred to Metal Organic Vapour Phase Epitaxy (MOVPE) chamber which consists of single-wafer lamp heated reactor with controlled gas flow rates. The sapphire substrate was heated until growth temperature $\left(1000{ }^{\circ} \mathrm{C}\right)$ under high purity $\mathrm{N}_{2} / \mathrm{H}_{2}$ gas, $\mathrm{Mo}(\mathrm{CO})_{6}$ solid precursor and $\mathrm{H}_{2} \mathrm{~S}$ gas were sent to growth chamber. The total pressure of the growth chamber was kept at 20 torr and Mo flux of $8 \times 10^{-3}$ torr and an $\mathrm{H}_{2} \mathrm{~S} / \mathrm{Mo}$ ratio of $1.2 \times 10^{4}$. After deposition, the $\mathrm{MoS}_{2}$ samples were annealed under $\mathrm{H}_{2} \mathrm{~S}$ gas flow at $1000{ }^{\circ} \mathrm{C}$ for quality improvement.

Covalent Functionalization of $\mathrm{MoS}_{2}$ : A solution of 4-NBD was prepared in water (for concentration details see text). For spontaneous grafting of 4-NP radicals on $\mathrm{MoS}_{2}$ the 4-NBD solution was directly drop casted on freshly cleaved bulk $\mathrm{MoS}_{2}$ for a specific duration (details see text). For chemically activated 4-NP grafting on $\mathrm{MoS}_{2}$, 1:1 ratio of 4-NBD and KI solutions were added and immediately drop casted on $\mathrm{MoS}_{2}$ surface. The samples were rinsed by ethanol and DI water and dried in continuous Ar flow.

SPM Experiments. STM experiments were performed at room temperature $\left(20-22^{\circ} \mathrm{C}\right)$ using a PicoLE (Keysight) or Molecular Imaging STM system operating in constant-current mode. Imaging parameters are indicated in the figure captions and are denoted by $V_{\text {bias }}$ for the sample bias and $I_{\text {set }}$ for the tunnelling current. STM tips were prepared by mechanical cutting from Pt/Ir wire $(80 \% / 20 \%$, diameter $0.25 \mathrm{~mm}$ ). STM measurements performed at liquid solid interface were carried out using 1-phenyloctane as solvent.

AFM measurements were performed in air with a Multimode AFM with a Nanoscope VIII controller (Bruker) and a PicoSPM (5100, Agilent Technologies) in intermittent contact mode. Olympus silicon cantilevers (AC160TS-R3), with resonance frequency around $300 \mathrm{kHz}$ and spring constant around $26 \mathrm{~N} / \mathrm{m}$, were used. For nanoshaving experiments, AC240TS-R3 cantilevers with resonance frequency around $70 \mathrm{kHz}$ and spring constant around $2 \mathrm{~N} / \mathrm{m}$, were also used. In this method, molecules are removed by sweeping them away with the AFM tip, while the machine is operated in contact mode with a typical force of $20 \mathrm{nN}$. STM and AFM data analysis was performed using WSXM 5.0.2. ${ }^{45}$. The surface coverage measured in AFM was estimated using the flooding function of WSXM.

XPS: The XPS spectra were recorded on a Kratos Axis Supra X-ray Photoelectron Spectrometer employing a monochromatic Al Ka X-ray source ( $h v=1486.7 \mathrm{eV}, 5 \mathrm{~mA}$ (survey), $8 \mathrm{~mA}$ (HR) emission). The high-resolution scans were acquired with $20 \mathrm{eV}$ pass energy and survey scans were acquired using a pass energy of $160 \mathrm{eV}$. All the measurements were carried out at a base pressure of $1 \times 10^{-8} \mathrm{mbar}$ and under charge neutralization conditions using a low energy electron gun within the field of the magnetic lens. Binding energy was referenced to $M o 3 d_{5 / 2}$ at $229.14 \mathrm{eV}$ as given for $\mathrm{MoS}_{2}$ in the literature. ${ }^{46}$ All scans were acquired at normal emission.

Raman. Raman measurements were performed with Monovista CRS+ (S\&I GmbH) Confocal Raman Microscope. $532 \mathrm{~nm}$ He-Ne laser light was focused onto the sample surface through an objective (OLYMPUS, BX43 100x, N.A. 0.7). Raman scattering was collected with the same objective and directed to a Raman spectrograph (S\&I $\mathrm{GmbH}$ ) equipped with a cooled-charge coupled device (CCD) camera operated at $-100{ }^{\circ} \mathrm{C}(\mathrm{S} \& \mathrm{I} \mathrm{GmbH})$. The spectra were acquired for 10 seconds and repeated 6 times at the same spot. The filter wheel was kept at $2.5 \mathrm{OD}$ resulting in a laser power of $1.420 \mathrm{~W}$ on the surface. All of the measurements were carried out under ambient conditions and at room temperature.

Photoluminescence: Steady-state photoluminescence spectra were taken in an Edinburgh FLS980 spectrophotometer with an excitation wavelength of $450 \mathrm{~nm}$, and acquisition time of $1 \mathrm{~s}$.

\section{Conflicts of interest}

There are no conflicts to declare 


\section{Acknowledgements}

L.D., J.T., M.C.R.G. and S.D.F thank KU Leuven - internal funds (grant C14/19/079), and Research Foundation Flanders (FWO) (grant G081518N, G0A3220N) for financial support. M.C.R.G. thanks KU Leuven for a PDM fellowship. W.T. and S.E. thank FWO (grant G0A1219N), KU Leuven (grant C14/18/061), and the European Union's European Fund for Regional Development, Flanders Innovation \& Entrepreneurship, and the Province of West-Flanders (Accelerate3 project, Interreg Vlaanderen- Nederland program) for financial support.

\section{References}

1 S. Manzeli, D. Ovchinnikov, D. Pasquier, O. V. Yazyev and A Kis, Nat. Rev. Mater. , 2017, 2, 17033.

2 W. Choi, N. Choudhary, G. H. Han, J. Park, D. Akinwande and Y. H. Lee, Mater. Today, 2017, 20, 116-130.

3 A. Splendiani, L. Sun, Y. Zhang, T. Li, J. Kim, C.-Y. Chim, G. Galli and F. Wang, Nano Lett. , 2010, 10, 1271-1275.

4 K. F. Mak, C. Lee, J. Hone, J. Shan and T. F. Heinz, Phys. Rev. Lett. , 2010, 105, 136805.

5 L. Daukiya, J. Seibel and S. De Feyter, Adv. Phys.: X, 2019, 4, 1625723.

6 S. Niyogi, E. Bekyarova, M. E. Itkis, H. Zhang, K. Shepperd, J. Hicks, M. Sprinkle, C. Berger, C. N. Lau, W. A. de Heer, E. H. Conrad and R. C. Haddon, Nano Lett., 2010, 10, 4061-4066.

7 K. S. Mali, J. Greenwood, J. Adisoejoso, R. Phillipson and S. De Feyter, Nanoscale, 2015, 7, 1566-1585.

8 L. Daukiya, C. Mattioli, D. Aubel, S. Hajjar-Garreau, F. Vonau, E. Denys, G. Reiter, J. Fransson, E. Perrin, M.-L. Bocquet, C. Bena, A. Gourdon and L. Simon, ACS Nano, 2017, 11, 627-634.

9 R. A. Bueno, J. I. Martínez, R. F. Luccas, N. R. del Árbol, C. Munuera, I. Palacio, F. J. Palomares, K. Lauwaet, S. Thakur, J. M. Baranowski, W. Strupinski, M. F. López, F. Mompean, M. García-Hernández and J. A. Martín-Gago, Nat. Commun., 2017, 8, 15306.

10 C. Ataca and S. Ciraci, J. Phys. Chem. C, 2011, 115, 1330313311.

11 Q. Tang and D.-e. Jiang, Chem. Mater. , 2015, 27, 3743-3748.

12 S. Ippolito, A. Ciesielski and P. Samorì, Chem. Commun. , 2019, 55, 8900-8914.

13 D. Voiry, A. Goswami, R. Kappera, C. d. C. C. e. Silva, D. Kaplan T. Fujita, M. Chen, T. Asefa and M. Chhowalla, Nat. Chem., 2014, 7, 45-49.

14 M. Makarova, Y. Okawa and M. Aono, J. Phys. Chem. C, 2012, 116, 22411-22416.

15 X. Chen, N. C. Berner, C. Backes, G. S. Duesberg and A. R. McDonald, Angew. Chem. Int. Ed., 2016, 55, 5803-5808.

16 X. S. Chu, A. Yousaf, D. O. Li, A. A. Tang, A. Debnath, D. Ma, A. A. Green, E. J. G. Santos and Q. H. Wang, Chem. Mater., 2018 30, 2112-2128.

17 K. C. Knirsch, N. C. Berner, H. C. Nerl, C. S. Cucinotta, Z. Gholamvand, N. McEvoy, Z. Wang, I. Abramovic, P. Vecera, M Halik, S. Sanvito, G. S. Duesberg, V. Nicolosi, F. Hauke, A. Hirsch, J. N. Coleman and C. Backes, ACS Nano, 2015, 9, 60186030.

18 D. O. Li, X. S. Chu and Q. H. Wang, Langmuir, 2019, 35, 56935701.

19 Y. Park, S. Shin, Y. An, J.-G. Ahn, G. Shin, C. Ahn, J. Bang, J. Baik, Y. Kim, J. Jung and H. Lim, ACS Appl. Mater. Interfaces, 2020, 12, 40870-40878.

20 M. Vera-Hidalgo, E. Giovanelli, C. Navío and E. M. Pérez, J. Am. Chem. Soc. , 2019, 141, 3767-3771.

21 S. Presolski, L. Wang, A. H. Loo, A. Ambrosi, P. Lazar, V. Ranc, M. Otyepka, R. Zboril, O. Tomanec, J. Ugolotti, Z. Sofer and M. Pumera, Chem. Mater. , 2017, 29, 2066-2073.
22 X. Chen and A. R. McDonald, Adv. Mater., 2016, 28, 57385746.

23 A. Förster, S. Gemming, G. Seifert and D. Tománek, ACS Nano, 2017, 11, 9989-9996.

24 Q. Li, Y. Zhao, C. Ling, S. Yuan, Q. Chen and J. Wang, Angew. Chem. Int. Ed., 2017, 56, 10501-10505.

25 P. Allongue, M. Delamar, B. Desbat, O. Fagebaume, R. Hitmi, J. Pinson and J.-M. Savéant, J. Am. Chem. Soc., 1997, 119, 201207.

26 E. X. Yan, M. Cabán-Acevedo, K. M. Papadantonakis, B. S. Brunschwig and N. S. Lewis, ACS Mater. Lett., 2020, 2, 133139.

27 A. Mesnage, S. Esnouf, P. Jégou, G. Deniau and S. Palacin, Chem. Mater., 2010, 22, 6229-6239.

28 V. Mévellec, S. Roussel, L. Tessier, J. Chancolon, M. MayneL'Hermite, G. Deniau, P. Viel and S. Palacin, Chem. Mater., 2007, 19, 6323-6330.

29 M. C. Rodríguez González, A. Brown, S. Eyley, W. Thielemans, K. S. Mali, and S. De Feyter, Nanoscale, 2020, 12, 18782-18789

30 D. Kosynkin, T. M. Bockman and J. K. Kochi, J. Am. Chem. Soc., 1997, 119, 4846-4855.

31 Y. Xia, C. Martin, J. Seibel, S. Eyley, W. Thielemans, M. van der Auweraer, K. S. Mali and S. De Feyter, Nanoscale, 2020, 12, 11916-11926.

32 Mo, J.; El Kazzi, S.; Mortelmans, W.; Mehta, A. N.; Sergeant, S.; Smets, Q.; Asselberghs, I.; Huyghebaert, C. Nanotechnology, 2020, 31, 125604

33 B. M. Simons, J. Lehr, D. J. Garrett and A. J. Downard, Langmuir, 2014, 30, 4989-4996.

34 J. Greenwood, T. H. Phan, Y. Fujita, Z. Li, O. Ivasenko, W. Vanderlinden, H. Van Gorp, W. Frederickx, G. Lu, K. Tahara, Y. Tobe, H. Uji-i, S. F. L. Mertens and S. De Feyter, ACS Nano, 2015, 9, 5520-5535.

35 E. Bekyarova, S. Sarkar, S. Niyogi, M. E. Itkis and R. C. Haddon, J. Phys. D: Appl. Phys., 2012, 45, 154009.

36 S. Svensson, A. Naves de Brito, M. P. Keane, N. Correia and L. Karlsson, Phys. Rev. A, 1991, 43, 6441-6443.

37 D. Ganta, S. Sinha, and Richard T. Haasch, Surf. Sci. Spectra, 2014, 21, 19-27

38 A. Syari'ati, S. Kumar, A. Zahid, A. Ali El Yumin, J. Ye and P. Rudolf, Chem. Commun., 2019, 55, 10384-10387.

39 B. Chakraborty, H. S. S. R. Matte, A. K. Sood and C. N. R. Rao, J. Raman Spectrosc., 2013, 44, 92-96.

40 H. Li, Q. Zhang, C. C. R. Yap, B. K. Tay, T. H. T. Edwin, A. Olivier and D. Baillargeat, Adv. Funct. Mater., 2012, 22, 1385-1390.

41 S. Mouri, Y. Miyauchi and K. Matsuda, Nano Lett., 2013, 13, 5944-5948.

42 C. R. Ryder, J. D. Wood, S. A. Wells, Y. Yang, D. Jariwala, T. J. Marks, G. C. Schatz and M. C. Hersam, Nat. Chem., 2016, 8, 597-602.

43 H. G. Ji, P. Solís-Fernández, D. Yoshimura, M. Maruyama, T. Endo, Y. Miyata, S. Okada and H. Ago, Adv. Mater., 2019, 31, 1903613.

44 K. Hemanjaneyulu, J. Kumar and M. Shrivastava, IEEE Trans. Electron Devices, 2019, 66, 3224-3228.

45 I. Horcas, R. Fernández, J. M. Gómez-Rodríguez, J. Colchero, J. Gómez-Herrero and A. M. Baro, Review of Scientific Rev. Sci. Instrum., 2007, 78, 013705.

46 J. Kong, K. T. Park, A. C. Miller, and K. Klier, Surf. Sci. Spectra, $2000,7,69-74$ 